\title{
Design of Body Slip Angle Observer for Vehicle Stability Control
}

\author{
Liu Minghui ${ }^{1}$, Zhang Yongsheng ${ }^{1, a},{\text { Chu } \text { Liang }^{2} \text {, Wei Wenruo }}^{1}$ \\ Cai Jianwei ${ }^{2}$, Zhang Lei $^{2}$ \\ ${ }^{2}$ R\&D Center, China FAW Co., Ltd., Changchun, China \\ ${ }^{2}$ State Key Laboratory of Automobile Simulation and Control, Jilin University, Changchun, China \\ a Email: zhangyongsheng1@rdc.faw.com.cn
}

Keywords: Body Slip Angle; Lateral Velocity; Vehicle Stability Control; Observer

\begin{abstract}
Body slip angle is important for Vehicle Stability Control. Due to using sensors to measure body slip angle is very expensive, it is necessary to estimate it from other variables measured easily. A novel method based on non-linear robust observer is proposed for estimation body slip angle. Firstly, full-dimension observer designed based on yaw rate and lateral acceleration to estimate lateral velocity. And then, the body slip angle is estimated by using lateral velocity and longitudinal velocity. The proposed method is verified by offline simulation. The results demonstrate that this method has a better adaptability to different driving conditions and accuracy.
\end{abstract}

\section{Introduction}

In order to achieve the vehicle stability control, the yaw rate $\dot{\psi}$ and body slip angle $\beta$ are generally used as the control reference variables ${ }^{[1]}$. Yaw rate can be measured directly, but the body slip angle is estimated through the other vehicle motion state parameters, such as steering wheel angle, lateral acceleration, yaw-rate, etc ${ }^{[2]}$, because it is difficult to measure directly with low cost ${ }^{[3]}$. Therefore, looking for a kind of body slip angle estimation method with high precision and good real-time has become a key research problem of stability control.

Body slip angle estimation methods can be briefly summarized into two categories: 1) estimated by combinations of the different types of inertial sensors; 2) slip angle estimated by using the inertial sensor combined with vehicle dynamics model. According to previous research results and the second category, based on tire cornering stiffness online identification, a robust full-dimension observer for estimating vehicle lateral velocity is designed. The lateral velocity and longitudinal velocity are combined to estimate the body slip angle, and its schematic diagram is shown as Figure 1. It can be seen from Figure 1, the body slip angle estimation strategy is described as three parts: lateral velocity estimation, tire cornering stiffness estimation and body slip angle estimation.

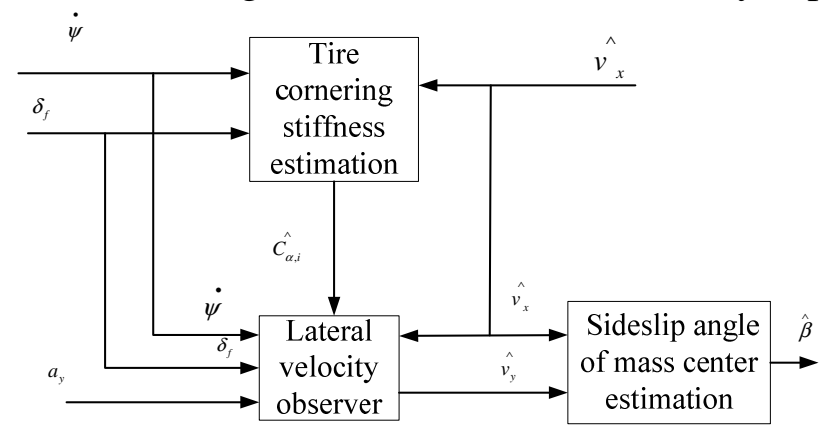

Fig 1 body slip angle estimates diagram

Lateral velocity estimation. In this paper, a robust full-order observer ${ }^{[4]}$ is used to design the car lateral velocity observer and the robustness of the observer gain.

Lateral velocity Observer design. Lateral velocity observer design adopts a simplified two-degree-of-freedom vehicle model:

$$
\begin{aligned}
& m \cdot \dot{v}_{y}=-m \cdot v_{x} \cdot \dot{\psi}+2 F_{y, f}+2 F_{y, r} \\
& J_{z} \cdot \ddot{\psi}=2 \cdot l_{a} \cdot F_{y, f}-2 \cdot l_{b} \cdot F_{y, r}+M_{z}
\end{aligned}
$$


Wherein the lateral force is expressed as:

$$
\begin{aligned}
& F_{y, i}=C_{\alpha, i} \alpha_{i} \quad i=f, r \\
& \alpha_{f}=\delta_{f}-\frac{v_{y}+l_{a} \dot{\psi}}{v_{x}} \\
& \alpha_{r}=\frac{l_{b} \dot{\psi}-v_{y}}{v_{x}}
\end{aligned}
$$

By formula (1) to (5), the equation of state of the system is as shown:

$$
\dot{x}=A x+B u
$$

Where:

$$
\begin{aligned}
& x=\left[\begin{array}{l}
v_{y} \\
\dot{\psi}
\end{array}\right], \quad u=\left[\begin{array}{c}
\delta_{f} \\
M_{z}
\end{array}\right] \\
& A=\left[\begin{array}{ll}
a_{11} & a_{12} \\
a_{13} & a_{14}
\end{array}\right]=\left[\begin{array}{cc}
-\frac{C_{\alpha, f}+C_{\alpha, r}}{m v_{x}} & \frac{l_{b} \cdot C_{\alpha, r}-l_{a} \cdot C_{\alpha, f}}{m v_{x}^{2}}-1 \\
\frac{l_{b} \cdot C_{\alpha, r}-l_{a} \cdot C_{\alpha, f}}{J_{z}} & -\frac{l_{b}^{2} \cdot C_{\alpha, r}+l_{a}^{2} \cdot C_{\alpha, f}}{J_{z} \cdot v_{x}}
\end{array}\right] \\
& B=\left[\begin{array}{ll}
b_{11} & b_{12} \\
b_{21} & b_{22}
\end{array}\right]=\left[\begin{array}{ll}
\frac{C_{\alpha, f}}{m \cdot v_{x}} & 0 \\
\frac{l_{a} \cdot C_{\alpha, f}}{J_{z}} & \frac{1}{J_{z}}
\end{array}\right]
\end{aligned}
$$

By the formula (6), the system in the vehicle driving process $\left(v_{x} \neq 0\right.$ ) is always considerable; When $v_{x}=0$, the vehicle stops and the system is not observable, then the lateral velocity estimation value is set to zero directly.

In the model, tire cornering stiffness is extremely an important parameter. The changes of the road surface friction coefficient and tire load will make it change and when the tire entering the nonlinear of $\mu-\lambda$ curve, cornering stiffness will change greatly. This change will make the model do not match the actual state of motion, which is unfavorable for the robustness and convergence of the designed observer. It must be online identification for the tire cornering stiffness, the identification method use least-squares estimation, estimation methods are as equation (7) to (10):

$$
\begin{aligned}
& \begin{array}{l}
y=x^{T} \theta \\
\text { Where: } y=v_{x} \\
\theta=C_{\alpha, f} \\
x=\left[\frac{\zeta \cdot\left(l_{a}+l_{b}\right) \cdot \delta_{s t}}{\dot{\psi} \cdot m \cdot\left(\zeta \cdot l_{a}-l_{b}\right)}-\frac{\left(l_{a}+l_{b}\right)^{2} \cdot \zeta}{m \cdot\left(\zeta \cdot l_{a}-l_{b}\right) \cdot v_{x}}\right]
\end{array}
\end{aligned}
$$

Tire cornering stiffness can be estimated using recursive least-squares method by taking equation (7) into the equation (8) to (10). Where: $\lambda_{R L S}$ is forgetting factor.

$$
\begin{aligned}
& \hat{\theta}(k)=\hat{\theta}(k-1)-\frac{P(k-1) \cdot x(k)}{\lambda_{R L S}+x^{T}(k) \cdot P(k-1) \cdot x(k)} \cdot e_{1}(k) \\
& e_{1}(k)=x^{T}(k) \cdot \hat{\theta}(k-1)-y(k) \\
& P(k)=\frac{1}{\lambda_{R L S}}\left\{P(k-1)-\frac{P(k-1) \cdot x(k) \cdot x^{T}(k) \cdot P(k-1)}{\lambda_{R L S}+x^{T}(k) \cdot P(k-1) \cdot x(k)}\right\}
\end{aligned}
$$

When constructing lateral velocity observer output equation, signals of ESP sensors for lateral acceleration and yaw rate are needed. These two signals have the following relationship: 
$a_{y}=\dot{v}_{y}+v_{x} \cdot \dot{\psi}$

Using the equation (6) and (11):

$a_{y}=a_{11} \cdot v_{y}+\left(a_{12}+v_{x}\right) \cdot \dot{\psi}+b_{11} \cdot \delta_{f}$

The system output equations can be formulated as:

$y=C x+D u$

Where, $y=\left[\begin{array}{c}a_{y} \\ \dot{\psi}\end{array}\right], C=\left[\begin{array}{cc}a_{11} & \left(a_{12}+v_{x}\right) \\ 0 & 1\end{array}\right], D=\left[\begin{array}{cc}b_{11} & 0 \\ 0 & 0\end{array}\right]$

The full-dimension state equation and its output equation of the system observer are as following:

$$
\begin{aligned}
& \dot{\hat{x}}=A \hat{x}+B u-K(\hat{y}-y) \\
& \hat{y}=C \hat{x}+D u
\end{aligned}
$$

Where, $K$-observer gain matrix; $\hat{x}$-the estimation of $x$; Estimation error of $v_{y}$ is $e=\hat{v}_{y}-v_{y}$. It should meet the equation:

$\dot{e}=(A-K C) e$

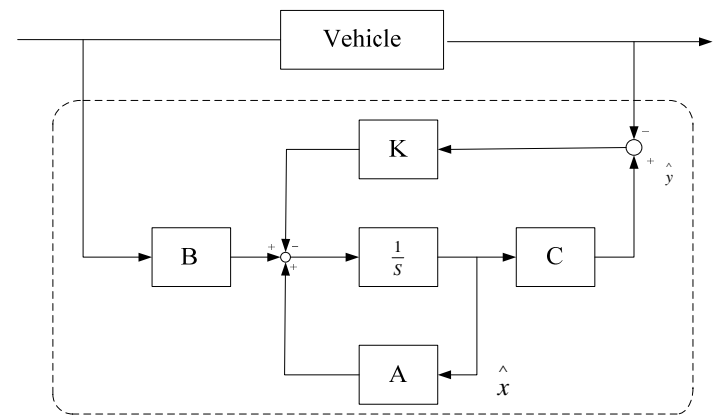

Fig 2 Schematic diagram of lateral speed full-order observer

The robust of the lateral velocity observer design. The value of the observer gain matrix $K$ is key to the robustness and stability of the observer. There are two aspects which need to be considered about:

Reduce the impact of the model error as much as possible to the lowest level;

Ensure that the characteristic values of the matrix $(A-K C)$ are located in the stable region.

Comparison of formula (12) and (13), the estimated value of ${ }^{v_{y}}$ :

$\dot{\hat{v}}_{y}=a_{11} \cdot \hat{v}_{y}+a_{12} \cdot \dot{\hat{\psi}}+b_{11} \cdot \delta-k_{11}(\dot{\hat{\psi}}-\dot{\psi})-k_{12}\left(\hat{a}_{y}-a_{y}\right)$

The actual value of $v_{y}$ with no error:

$\dot{v}_{y}=a_{11, r} \cdot v_{y}+a_{12, r} \cdot \dot{\psi}+b_{11, r} \cdot \delta$

Where, ${ }^{a_{11, r}}, a_{12, r}, b_{11, r}$ represent the real values of the model parameters excluding any error.

Using the equation (16) and (17):

$$
\begin{aligned}
& \dot{\hat{v}}_{y}-\dot{v}_{y}=a_{11} \cdot\left(1-k_{12}\right) \cdot\left(\hat{v}_{y}-v_{y}\right)+\left(1-k_{12}\right) \cdot\left(a_{11}-a_{11, r}\right) \cdot v_{y} \\
& +\left(1-k_{12}\right) \cdot\left(b_{11}-b_{11, r}\right) \cdot \delta+\left(1-k_{12}\right) \cdot\left(a_{12}-a_{12, r}\right) \cdot \dot{\psi} \\
& +\left[a_{12} \cdot\left(1-k_{12}\right)-k_{12} \cdot v_{x}-k_{11}\right] \cdot(\dot{\hat{\psi}}-\dot{\psi})
\end{aligned}
$$

As for equation (18), in order to increase the robustness of the lateral velocity observer, $\left(\dot{\hat{v}}_{y}-\dot{v}_{y}\right) \rightarrow 0$ should be ensured. Considering $(\dot{\hat{\psi}}-\dot{\psi}) \rightarrow 0$, the following equation must be fulfilled:

$$
1-k_{12}=0 \Leftrightarrow k_{12}=1
$$

So,based on pole placement and robustness, the value of gain matrix $K$ is shown as ${ }^{[6]}$ : 


$$
K=\left[\begin{array}{cc}
\frac{p_{1} \cdot p_{2} \cdot\left(a_{11}+v_{x}\right)}{a_{11} \cdot a_{22}-a_{21}^{2}-a_{21} \cdot v_{x}} & 1 \\
-p_{1}-p_{2} & \frac{a_{22}}{a_{21}+v_{x}}
\end{array}\right]
$$

Where, ${ }^{p_{1}}$ and ${ }^{p_{2}}$ are poles of the observer, its values are restricted by:

1) $p_{1} 、 p_{2}$ must be negative real number, otherwise observer is unstable;

2) when $p_{1} 、 p_{2}$ absolute values are large, the response of the observer is fast but anti-jamming is weaker;

3) when $p_{1}, p_{2}$ absolute values are small, the response of the observer is slow but anti-jamming is stronger.

Based on the above conditions, it meets the requirements of response speed and has a good anti-jamming capability by adjusting the values of $p_{1} 、 p_{2}$ in the different operating conditions.

Final estimation of body slip angle. The longitudinal velocity $\hat{v}_{x}$ and the lateral velocity $\hat{v}_{y}$ are got through the longitudinal and lateral velocity observer. body slip angle is formulated as the following equation:

$$
\hat{\beta}=\arctan \left(\frac{\hat{v}_{y}}{\max \left(\hat{v}_{x}, v_{c}\right)}\right)
$$

Where, ${ }^{v_{c}}$ represents a constant greater than zero to prevent zero divided in the calculation process. Finally body slip angle is got after filtering and compensation.

\section{Simulation}

The control algorithm is established in the MATLAB/Simulink, and verified by simulation. Figure 3, 4, and 5 show most simulation results under different working conditions. The actual value represents the measured value of the sensor in real vehicle simulation. The measured value is used as an accurate value for the estimation. The estimated value is the result of this algorithm's estimated value. As shown in figure 3, 4, and 5, estimated value and actual value have a good consistent tendency with little error. It can be seen that the designed estimation method can effectively estimate body slip angle in different conditions.

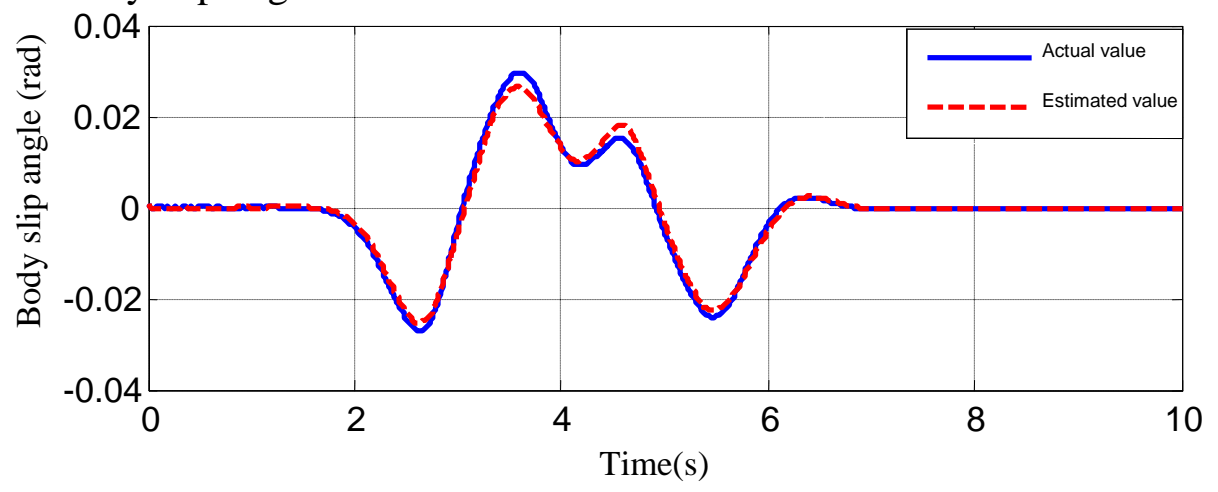

Fig 3 The simulation results of double lane on high adhesion road 


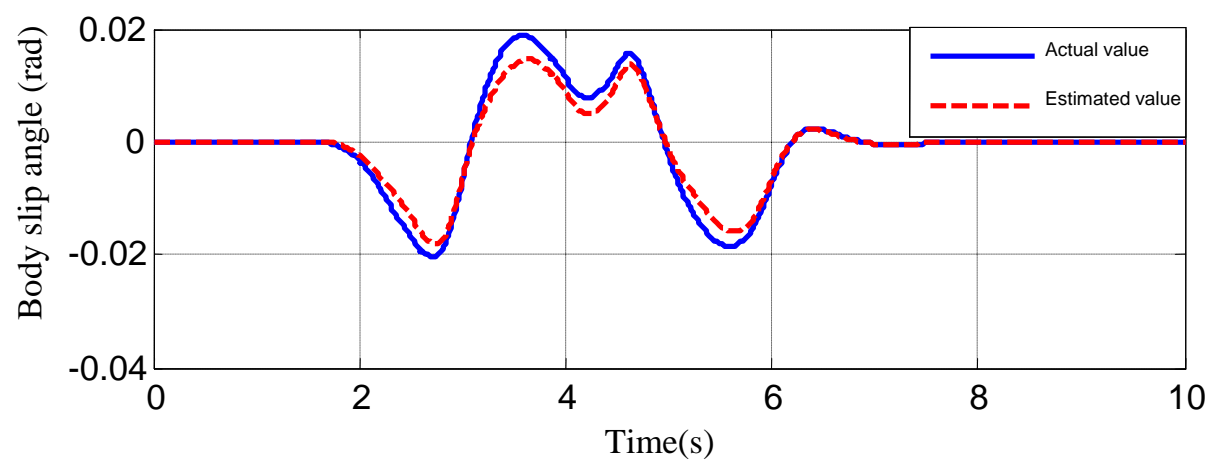

Fig 4 The simulation results of double lane on low-high adhesion road

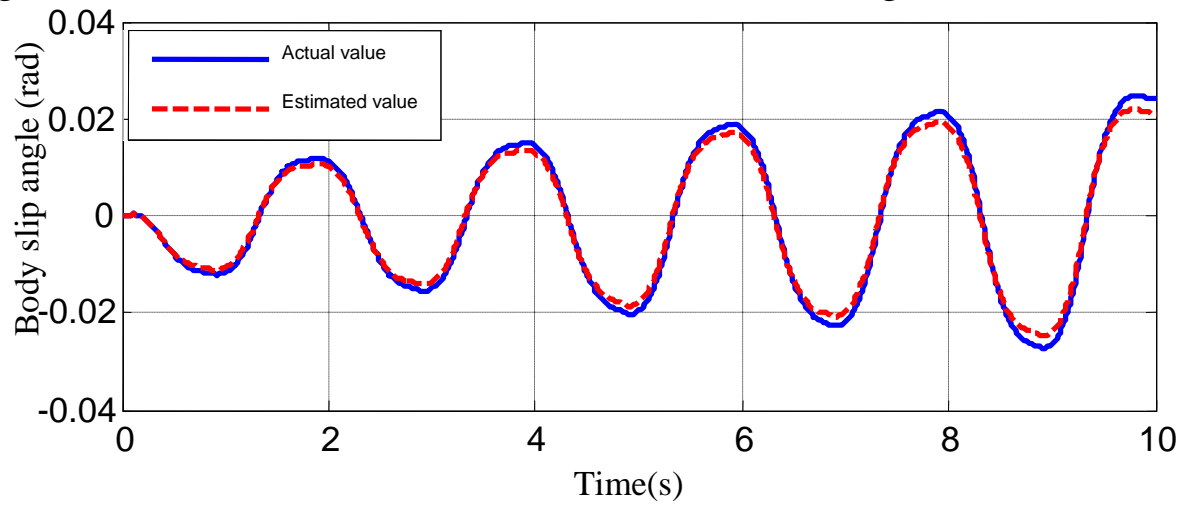

Fig 5 The simulation results of the snake-like conditions

\section{Results}

This paper is in the light of the problem that vehicle body slip angle is difficult to measure directly with low cost; A nonlinear robust observer for body slip angle was designed by using inertial sensors and vehicle dynamics model. Through MATLAB/Simulink modeling and simulating, the design of the algorithm was tested and verified. The results show that the observer has a high level precision, and has a better adaptability to different working conditions as well.

\section{Reference}

[1] Van Zanten A T. Evolution of electronic control systems for improving the vehicle dynamic behavior[C] .AVEC’02. 2002, :4481-4488.

[2] Hu Ai Jun, Shi Guo Biao, Lin Yi. Estimation of Automobile Steering State Parameters [J]. Journal of Highway and Transportation Research and Development, 2008, 25(10): 150-158.

[3] Fu Hao. Research on Sideslip Angle Estimation and Control Algorithm for Vehicle Electronic Stability System [D]. Jilin University, Changchun, China., 2008.

[4] Toshihiro Hiraoka, Hiromitsu Kumamoto, Osamu Nishihara. Side slip angle estimation and active front steering system based on lateral acceleration data at centers of percussion with respect to front/rear wheels[C]. JSAE, 20025539.

[5] Florin Moldoveanu, Mihai Cernat, Marius Georgescu, Dan Floroian. Vehicle Body Side Slip Angle Observer[C]. SAE: 2004-34-0016.

[6] Aoki, Y., Uchida, T., Hori, Y. Experimental demonstration of body slip angle control based on a novel linear observer for electric vehicle[C]. IECON, 2005, 250-257. 